\title{
Apatinib is effective for treatment of advanced hepatocellular carcinoma
}

\author{
Yinlong Kong ${ }^{1}$, Lin Sun ${ }^{1}$, Zhenyu Hou ${ }^{1}$, Yongqiang Zhang ${ }^{1}$, Ping Chen ${ }^{1}$, Yunlong \\ Cui $^{1}$, Xiaolin Zhu ${ }^{1}$, Tianqiang Song ${ }^{1}$, Qiang Li $^{1}$, Huikai Li ${ }^{1}$, Ti Zhang ${ }^{1}$ and Lunxiu \\ Qin ${ }^{2,3}$ \\ ${ }^{1}$ Tianjin Medical University Cancer Institute and Hospital, Key Laboratory of Cancer Prevention and Therapy, National Clinical \\ Research Center for Cancer, Tianjin 300060, China \\ ${ }^{2}$ Department of General Surgery, Huashan Hospital \& Cancer Metastasis Institute, Fudan University, Shanghai 200040, China \\ ${ }^{3}$ Cancer Research Center, Institute of Biomedical Science, Fudan University, Shanghai 200032, China \\ Correspondence to: Ti Zhang, email: zhangti@tjmuch.com \\ Huikai Li, email: tjchlhk@126.com \\ Lunxiu Qin, email: qinlx99@vip.163.com
}

Keywords: apatinib; hepatocellular carcinoma; vascular endothelial growth factor receptor

Received: May 24, $2017 \quad$ Accepted: September 21, $2017 \quad$ Published: November 06, 2017

Copyright: Kong et al. This is an open-access article distributed under the terms of the Creative Commons Attribution License 3.0 (CC BY 3.0), which permits unrestricted use, distribution, and reproduction in any medium, provided the original author and source are credited.

\section{ABSTRACT}

As treatment options for hepatocellular carcinoma (HCC) are currently limited, we evaluated the efficacy and safety of oral apatinib, a VEGFR-2 inhibitor, on patients with advanced HCC. Twenty-two patients from Tianjin Medical University Cancer Institute and Hospital were enrolled for evaluation. Apatinib was administered at $500 \mathrm{mg} /$ day or $250 \mathrm{mg} /$ day continuously. Clinical endpoints were time to disease progression (TTP), overall survival (OS), and safety. The median TTP of treated patients was 10.4 months (95\% CI $3.4-17.5)$. At the last follow-up, $50 \%$ patients had survived longer than $\mathbf{1 1 . 4}$ months from the first dose. Complete response (CR), partial response (PR), stable disease (SD), and progressive disease (PD) rates were $0 \%, 40.9 \%, 40.9 \%$, and $18.2 \%$, respectively. The most common apatinib-related adverse events were hand-foot skin reaction (HFSR) $(81.8 \%)$ and diarrhea $(77.3 \%)$. Hypertension ( $27.3 \%)$ and HFSR (13.6\%) were the most frequent grade $3 / 4$ adverse events. In summary, results of this small study indicate that apatinib is well tolerated and extremely effective for the treatment of advanced HCC. It is therefore imperative to design and carry out well-controlled clinical trials to confirm its efficacy.

\section{INTRODUCTION}

Hepatocellular carcinoma (HCC) is a major public health problem, ranking fourth in incidence and third in cancer-related mortality in China [1]. The main reason of HCC's high mortality rate is the lack of effective detection in the early stage. Consequently, it is estimated that $70 \%$ $85 \%$ of patients are diagnosed in the advanced stage [2]. For these patients, current treatment options show only modest efficacy.

Sorafenib is the standard treatment for advanced HCC. As a multi-target kinase inhibitor, sorafenib can suppress HCC cell proliferation by inhibiting the Raf/ MEK/ERK signaling pathway. In addition, sorafenib blocks angiogenesis in HCC by inhibiting VEGFR and PDGFR [3]. The phase III SHARP [4] and ORIENTAL [5] clinical trials indicated that sorafenib prolongs overall survival in advanced HCC patients. However, the partial response rates of HCC to sorafenib were only $2 \%$ and $3.3 \%$, and the extended survival was limited to 2.8 and 2.3 months, respectively. Furthermore, the occurrence of drug resistance and the expensive costs of treatment greatly limit the clinical application of sorafenib. 
Many target drugs against advanced $\mathrm{HCC}$ continue to emerge from clinical trials, but none of them have so far proved to be superior to sorafenib. Recently, apatinib, an oral small molecule anti-angiogenesis agent, came to light due to its positive effect on advanced gastric cancer $[6,7]$ and HCC [8]. Apatinib exerts wide-ranging tumor inhibition both in vitro and in vivo by binding VEGFR-2 specifically $[9,10]$, and has been approved for the third-line treatment of gastric adenocarcinoma and gastroesophageal junction adenocarcinoma after phase II/III clinical trials [6, 7]. Moreover, numerous clinical studies of apatinib on solid tumors, such as non-small cell lung cancer, breast cancer, and HCC, have shown promising clinical efficacy $[8,11-14]$.

Considering the limitations of sorafenib, as well as the experience derived from a small group of advanced HCC patients treated with apatinib at our institute, we carried out a clinical study to explore the efficacy and safety of apatinib in advanced HCC.

\section{RESULTS}

This study was carried out in patients who were sorafenib-resistant or could not afford the costs of sorafenib and were advised to receive apatinib. Written informed consent was obtained from 22 enrolled patients (19 males and 3 females), aged 32 to 77 years old (average, 54.3 years old). Among them, 21 patients had chronic hepatitis B virus infection and 13 had received antiviral therapy; 11 patients had postoperative recurrence and metastasis; 11 presented with macro-vascular invasion; 16 showed intrahepatic metastasis; 15 received previous or concomitant trans-arterial chemoembolization (TACE); and 21 had abnormally high serum alpha-fetoprotein (AFP) (Table 1).

By the end of the last follow-up (July 20th, 2017), the median time to disease progression (mTTP) for these 22 patients was 10.4 months (95\% CI 3.4-17.5). Seven patients died and 11 patients survived more than 11.4 months (Figure 1). Efficacy evaluation showed that complete response (CR), partial response (PR), stable disease (SD), and progressive disease (PD) were $0 \%(0 / 22), 40.9 \%(9 / 22), 40.9 \%(9 / 22)$ and $18.2 \%$ (4/22), respectively. The objective response rate (ORR) was $40.9 \%(9 / 22)$. Univariate analysis showed that the following factors may affect TTP: Albumin (ALB) $\geq 40.0$ $\mathrm{g} / \mathrm{L}(\mathrm{p}=0.013) ;$ Total Bilirubin (TBIL) $<21.0 \mu \mathrm{mol} / \mathrm{L}(\mathrm{p}$ $=0.031)$; Platelet $(\mathrm{PLT})<125 \times 10^{9} / \mathrm{L}(\mathrm{p}=0.031)$; and a decrease in AFP $(p=0.012)$. Multivariate analysis showed that TTP may be affected by: PLT $<125 \times 10^{9} / \mathrm{L}$ [HR 7.753 (95\% CI 1.160-51.823); $\mathrm{p}=0.03]$; and a decrease in AFP [HR 0.117 (95\% CI 0.30-0.692); $\mathrm{p}=0.018$ ] (Table 1 and Figure 2). After administration of apatinib, a reduction in AFP levels (i.e. AFP response) occurred in 14 cases, with 7 cases registering a reduction by half or more. Moreover, among these 7 cases, 6 were defined as PR with
AFP reaching normal level in 2 cases. Typical imaging changes in patients with advanced $\mathrm{HCC}$ receiving apatinib treatment are shown in Figure 3 and in Supplementary Figures.

Adverse events in the 22 patients mainly consisted of hand-foot skin reaction (HFSR) (81.8\%), diarrhea (77.3\%), hypertension $(63.6 \%)$, fatigue $(59.1 \%)$, hoarseness $(54.5 \%)$, and nausea $(50 \%)$. Grade 3 or 4 drug-related adverse events mainly included hypertension (27.3\%), HFSR (13.6\%), and thrombocytopenia (9.1\%). One case of upper gastrointestinal bleeding (in a patient receiving combined radiotherapy for bone metastasis) and 2 cases of cerebral hemorrhage were also recorded among patients with poor hypertension control. Because of hypertension, HFSR, diarrhea, and fatigue, 8 patients interrupted treatment, and another 6 required dose reduction. Meanwhile, 2 patients were withdrawn from treatment due to cerebral hemorrhage (Table 2).

\section{DISCUSSION}

Systemic treatment of advanced HCC remains a clinical challenge due to lack of effective drugs. Many first- and second-line agents have been tested in recent years, but the results were disappointing [15]. Currently, sorafenib is the only molecular targeted agent for advanced HCC that has received FDA approval after showing effectiveness in clinical trials. However, drug resistance and expensive treatment costs limit the application of sorafenib in the clinic.

The aim of the present study was to assess the efficacy and safety of daily administration of apatinib in the treatment of advanced HCC. In our patient cohort, mTTP (10.4 months) and ORR (40.9\%) were much better than expected. As a matter of fact, results from the SHARP study (mTTP: 5.5 months; ORR: 2\%) [4] and the ORIENTAL study (mTTP: 2.8 months; ORR: $3.3 \%$ ) [5] lagged far behind our own. Because less than half of the patients died by the end of the last follow-up, this study failed to obtain mOS. However, half of the 22 patients evaluated survived over 11.4 months, an outcome also superior to that of the ORIENTAL study (mOS: 6.5 months).

In the present study, the first patient who received apatinib treatment was an elderly female who had been treated with sorafenib and regorafenib (clinical trial) because of metastasis and recurrence after HCC surgery. Drug resistance, multiple liver lesions, and multiple metastases to the abdominal cavity and lung developed in this patient during sorafenib and regorafenib treatment. This PR case attracted our attention because, surprisingly, her serum AFP level dropped from 899.2 $\mu \mathrm{g} / \mathrm{L}$ to $74.03 \mu \mathrm{g} / \mathrm{L}$ after administration of apatinib for only 4 weeks. Moreover, 12 weeks later, not only her AFP values were within normal range, but also the tumors in her liver, abdominal cavity, and lung had shrank 
Table 1: Univariate and multivariate analyses of time to disease progression in advance HCC patients under the treatment of apatinib

\begin{tabular}{|c|c|c|c|c|c|c|}
\hline \multirow[b]{2}{*}{ Variable } & \multirow[b]{2}{*}{ N (\%) } & \multirow[b]{2}{*}{ mTTP(month) } & \multicolumn{2}{|c|}{ Univariate analysis } & \multicolumn{2}{|c|}{ Multivariate analysis } \\
\hline & & & $95 \% \mathrm{CI}$ & p Value & $\begin{array}{c}\text { Relative risk } \\
(95 \% \mathrm{CI})\end{array}$ & p Value \\
\hline Median age(Year) & $54.3(32-77)$ & & & 0.298 & & \\
\hline$<65$ & 22 & 7.1 & $2.1-12.0$ & & & \\
\hline$\geq 65$ & 4 & 10.6 & - & & & \\
\hline Gender & & & & 0.632 & & \\
\hline Male & 19 & 10.4 & $2.7-18.1$ & & & \\
\hline Female & 3 & 4.7 & - & & & \\
\hline ECOG- PS & & & & 0.944 & & \\
\hline 0 & 3 & 10.4 & - & & & \\
\hline 1 & 18 & 7.0 & $2.4-11.7$ & & & \\
\hline 2 & 1 & 10.6 & 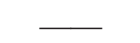 & & & \\
\hline $\mathrm{HBs}$ Ag & & & & 0.268 & & \\
\hline$(+)$ & 21 & 10.4 & $4.7-10.9$ & & & \\
\hline$(-)$ & 1 & - & - & & & \\
\hline Antivirus treatment & & & & 0.916 & & \\
\hline No & 8 & 10.6 & $10.3-10.9$ & & & \\
\hline Yes & 13 & 7.0 & $4.0-10.2$ & & & \\
\hline Combined TACE & & & & 0.096 & & \\
\hline NO & 7 & 10.9 & $3.3-18.4$ & & & \\
\hline Yes & 15 & 7.0 & $0.2-17.5$ & & & \\
\hline Relapse & & & & 0.910 & & \\
\hline NO & 11 & 10.9 & $0.4-21.4$ & & & \\
\hline Yes & 11 & 10.4 & $3.7-17.2$ & & & \\
\hline PVTT & & & & 0.494 & & \\
\hline NO & 11 & 10.9 & $3.5-17.3$ & & & \\
\hline Yes & 11 & 10.4 & $5.1-16.7$ & & & \\
\hline Metastasis & & & & 0.162 & & \\
\hline NO & 6 & 13.6 & $1.5-20.2$ & & & \\
\hline Yes & 16 & 10.4 & $3.4-17.5$ & & & \\
\hline Child-Pugh & & & & 0.087 & & \\
\hline A & 17 & 7.1 & $3.6-10.5$ & & & \\
\hline B & 5 & 10.9 & $10.4-11.3$ & & & \\
\hline ALT(U/L) & $47.2(15.0-236.0)$ & & & 0.641 & & \\
\hline$\geq 40$ & 9 & 4.7 & $3.4-17.5$ & & & \\
\hline$<40$ & 13 & 10.4 & $3.6-17.3$ & & & \\
\hline
\end{tabular}




\begin{tabular}{|c|c|c|c|c|c|c|}
\hline \multirow[b]{2}{*}{ Variable } & \multirow[b]{2}{*}{$\mathbf{N}(\%)$} & \multirow[b]{2}{*}{ mTTP(month) } & \multicolumn{2}{|c|}{ Univariate analysis } & \multicolumn{2}{|c|}{ Multivariate analysis } \\
\hline & & & $95 \% \mathrm{CI}$ & p Value & $\begin{array}{l}\text { Relative risk } \\
(95 \% \mathrm{CI})\end{array}$ & p Value \\
\hline $\operatorname{AST}(\mathrm{U} / \mathrm{L})$ & $64.2(24.0-247.0)$ & & & 0.707 & & \\
\hline$\geq 40$ & 15 & 7.0 & $1.7-12.4$ & & & \\
\hline$<40$ & 7 & 10.4 & $0.4-20.5$ & & & \\
\hline $\mathrm{ALP}(\mathrm{U} / \mathrm{L})$ & $146.4(25.0-293.0)$ & & & 0.704 & & \\
\hline$\geq 125$ & 13 & 10.6 & $0-22.0$ & & & \\
\hline$<125$ & 9 & 7.1 & $4.6-9.6$ & & & \\
\hline $\operatorname{ALB}(\mathrm{g} / \mathrm{L})$ & $38.0(26.0-53.1)$ & & & 0.013 & & \\
\hline$\geq 40.0$ & 10 & 5.8 & $2.3-9.4$ & & & \\
\hline$<40.0$ & 12 & 10.9 & $10.4-11.3$ & & & \\
\hline $\operatorname{TBIL}(\mu \mathrm{mol} / \mathrm{L})$ & $23.9(9.9-72.4)$ & & & 0.031 & & \\
\hline$\geq 21.0$ & 11 & 10.9 & $2.0-19.8$ & & & \\
\hline$<21.0$ & 11 & 5.8 & $3.4-17.5$ & & & \\
\hline $\mathrm{DBIL}(\mu \mathrm{mol} / \mathrm{L})$ & $7.4(2.0-39.8)$ & & & 0.838 & & \\
\hline$\geq 3.4$ & 14 & 10.4 & $0-21.1$ & & & \\
\hline$<3.4$ & 8 & 7.1 & $4.4-9.7$ & & & \\
\hline $\operatorname{HGB}(\mathrm{g} / \mathrm{L})$ & $138.7(87.0-185)$ & & & 0.719 & & \\
\hline$\geq 130.0$ & 16 & 7.1 & $3.6-10.5$ & & & \\
\hline$<130.0$ & 6 & 10.6 & - & & & \\
\hline $\operatorname{PLT}\left(10^{9} / \mathrm{L}\right)$ & $147.8(40.0-410.0)$ & & & 0.031 & $\begin{array}{c}7.753(1.160- \\
51.823)\end{array}$ & 0.035 \\
\hline$\geq 125$ & 13 & 10.6 & $3.4-17.5$ & & & \\
\hline$<125$ & 9 & 4.7 & $3.5-5.9$ & & & \\
\hline $\mathrm{AFP}>\mathrm{ULN}$ & 21 & & & & & \\
\hline AFP reduction & & & & 0.012 & $\begin{array}{c}0.117(0.20- \\
0.692)\end{array}$ & 0.018 \\
\hline AFP reduction $(+)$ & 14 & 10.6 & $2.6-18.6$ & & & \\
\hline AFP reduction (-) & 7 & 3.6 & $0.2-7.2$ & & & \\
\hline
\end{tabular}

ECOG PS=Eastern Cooperative Oncology Group Performance Status. BCLC=Barcelona Clinic Liver Cancer. $\mathrm{HBV}=$ hepatitis $\mathrm{B}$ virus. $\mathrm{HCV}=$ hepatitis $\mathrm{C}$ virus. $\mathrm{TACE}=$ trans arterial chemoembolization. $\mathrm{PVTT}=$ portal vein tumor thrombus. ULN, upper limit of normal.

or even disappeared. Noteworthily, only $7 / 299$, and $5 / 150$ sorafenib-treated patients in the SHARP and the ORIENTAL studies, respectively, had PR. Although the present data are not directly comparable with these two clinical studies, the fact that 9 out of 22 apatinib-treated patients in our study showed PR is very impressive and extremely inspiring.

Preclinical animal experiments and clinical trials showed that apatinib-related adverse events were dose dependent $[9,16]$. In this study, we mainly used apatinib at $250 \mathrm{mg} /$ day, which is markedly lower than the previously tested doses of $750-850 \mathrm{mg} /$ day [14]. In fact, patients who were initially treated at $500 \mathrm{mg} /$ day suffered from serious adverse events and were switched to $250 \mathrm{mg}$ /day. In general, patients receiving this low dose treatment had fewer and milder adverse events and achieved a good response. We speculate that this may be related to the background of liver disease in patients with HCC. Most patients with advanced HCC present also with chronic hepatitis or liver cirrhosis, and the poor hepatic functional reserve of these patients may affect the metabolism of apatinib. In addition, the general condition of patients with 

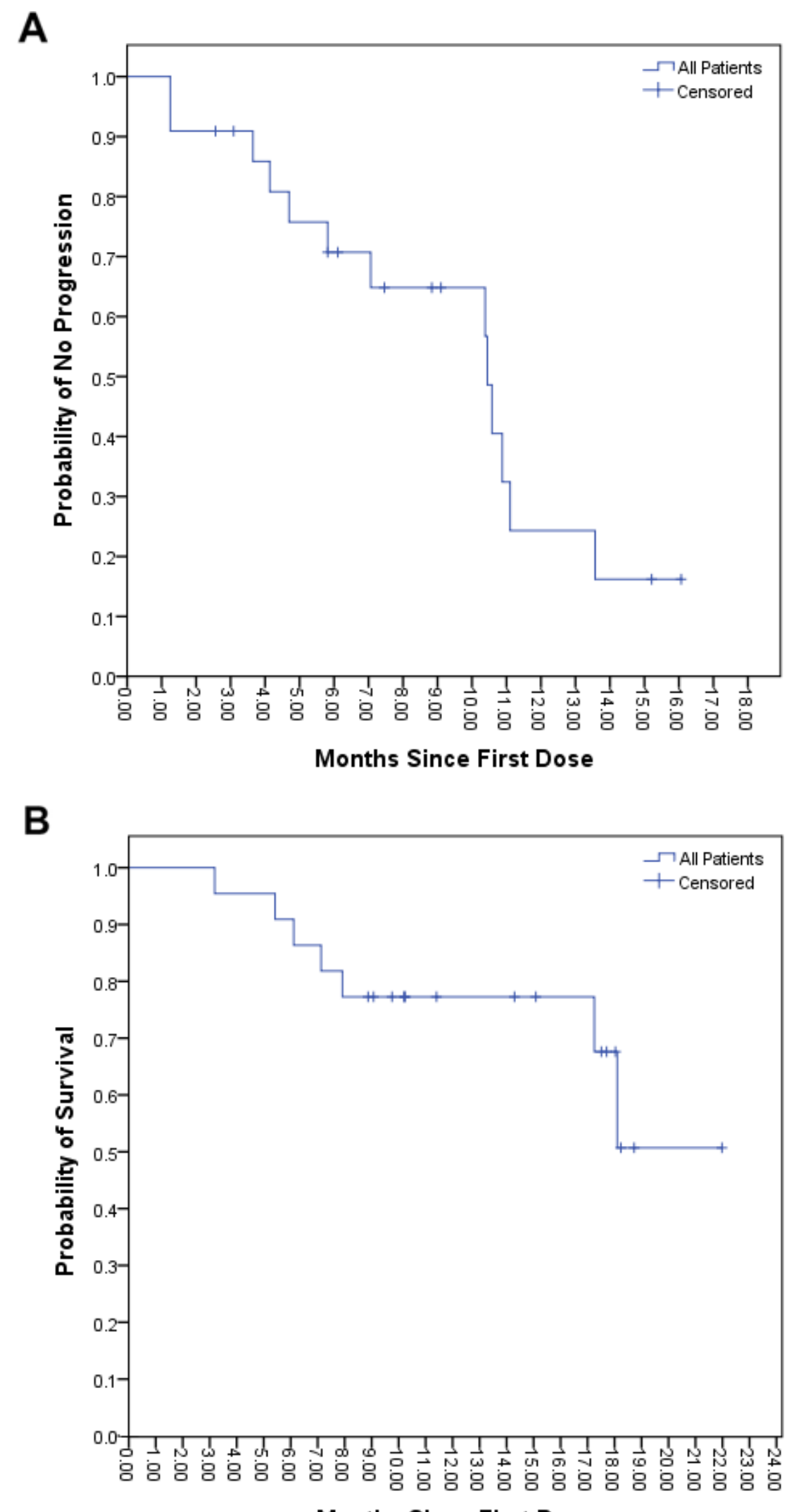

Figure 1: Kaplan-Meier Analysis of Time to Progression and Overall Survival. For 22 HCC patients receiving apatinib, the median time to progression was 10.4 months (Panel A). The median overall survival is not available, because only 7 patients had died by the end of observation. However, half of the 22 patients survived more than 11.4 months at this time point (Panel B). 
advanced HCC is not very good, which may further reduce the tolerance to apatinib-induced adverse events.

A phase II study showed that apatinib provides a potential survival benefit in HCC patients, and 850 $\mathrm{mg}$ /day or $750 \mathrm{mg}$ /day were the recommended doses for further clinical studies [14]. In our study, however, significant success was achieved using a relatively low dose of apatinib. Moreover, we noticed that none of the
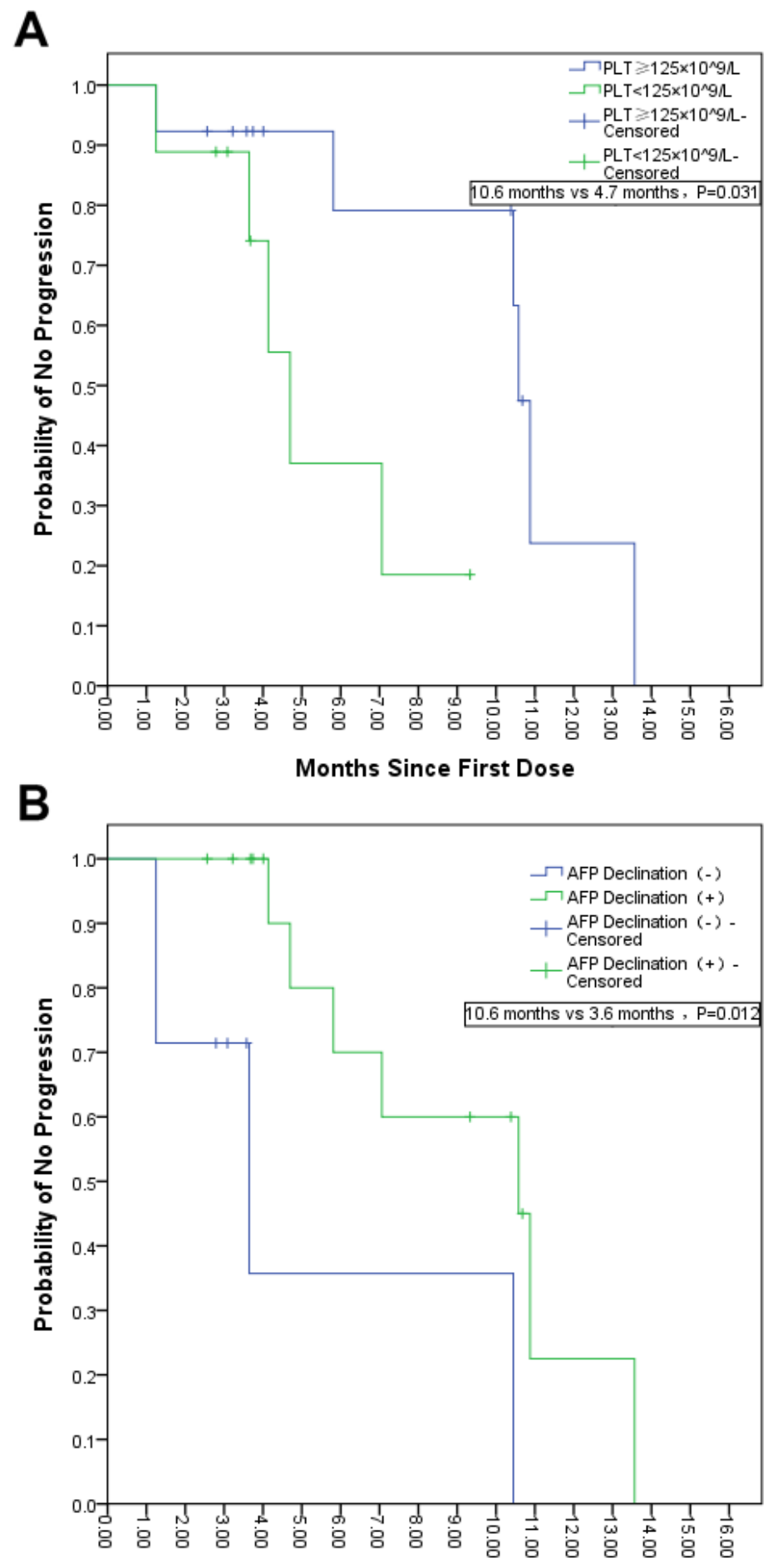

Months Since First Dose

Figure 2: Kaplan-Meier Analysis of the Time to Progression of Selected Subgroups. Multivariate analysis showed that platelet count and AFP reduction are associated with mTTP. The mTTP was only 4.7 months in patients with decreased platelet count $\left(<125 \times 10^{9} / \mathrm{L}\right)$, compared to 10.6 months in patients without decreased platelet count $\left(\geq 125 \times 10^{9} / \mathrm{L}\right)$ (Panel A). For patients with decreased AFP, mTTP was 10.6 months, compared to 3.6 months in patients without AFP response (Panel B). 
6 patients in this cohort initially treated with $500 \mathrm{mg} /$ day apatinib could tolerate this dose for more than 3 months. Nevertheless, our study showed that grade 3 or 4 adverse events may occur with low-dose apatinib, similarly to what has been reported for this drug in other tumor therapies $[10,11,17]$. Unlike the sorafenib study, our data did not indicate a correlation between efficacy and adverse events of apatinib. However, due to the limited sample size, this is not a definitive conclusion.

Considering the potential benefits of interventional therapy in HCC treatment, we analyzed the efficacy of apatinib treatment alone or combined with interventional therapy. The results indicated that the effect of interventional therapy did not increase or decrease the efficacy of apatinib. Similarly, the SPACE study showed that sorafenib did not increase the efficacy of TACE in middle stage HCC patients [18]. It is worth noting that 4 patients in our study received interventional therapy as the first choice. Due to ineffectiveness of treatment (PD), these patients were then treated with apatinib. Among them, 3 achieved PR and one reached SD. These results further suggest that apatinib may be efficacious in the adjuvant setting.

AFP is considered an important marker in the diagnosis and treatment of HCC. Chan et al. [19] and Shao et al. [20] defined the AFP response as a $20 \%$ reduction in serum AFP levels after treatment. Data have shown that an AFP decrease is a good prognostic surrogate in patients with HCC after radiofrequency ablation [21] or sorafenib treatment [22], whereas an early increase in AFP predicted unfavorable clinical outcomes in patients with advanced HCC treated with sorafenib [23]. Our results suggest that
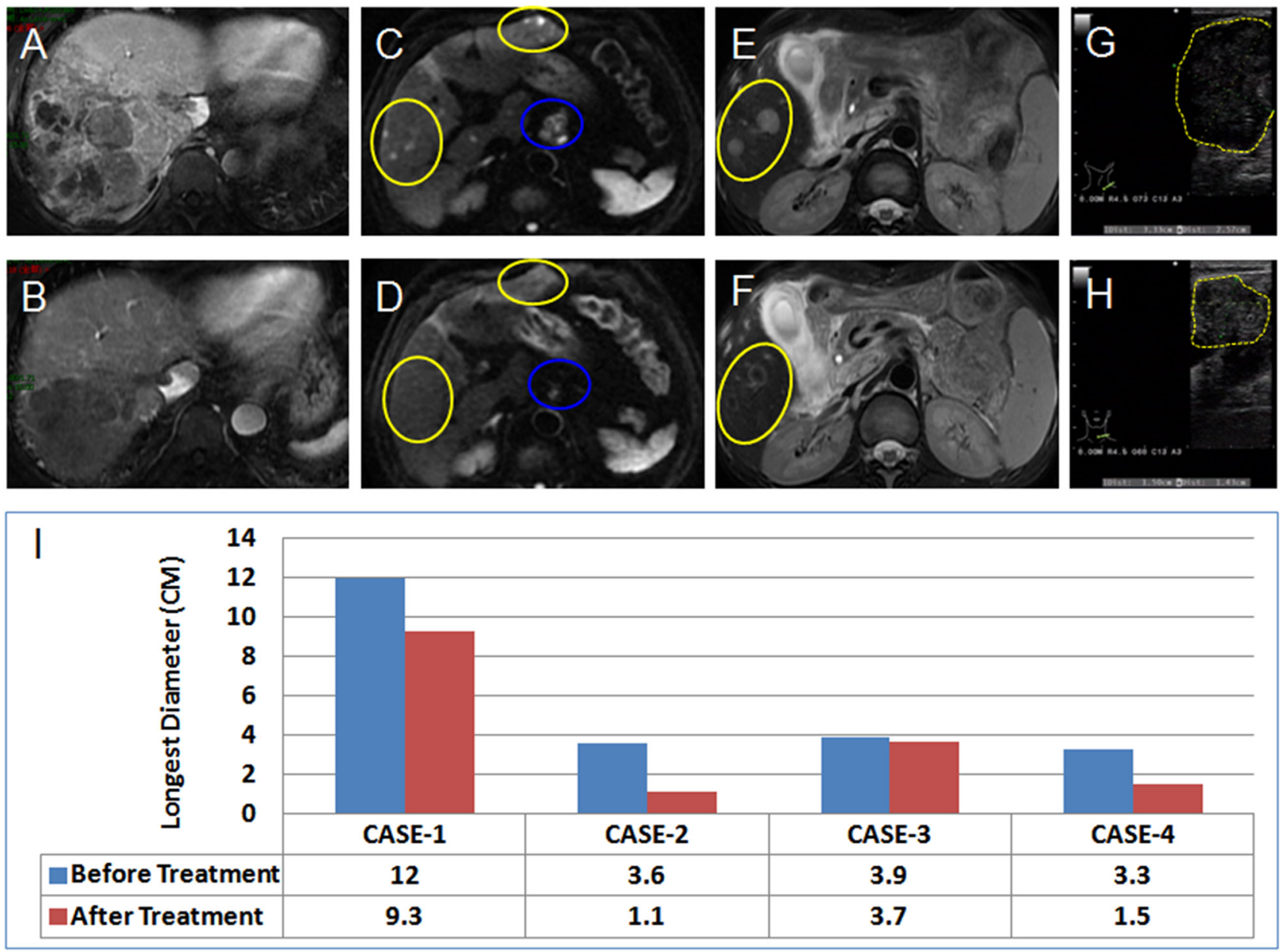

Figure 3: Typical Imaging Changes in Advanced HCC Patients Treated with Apatinib. Tyical imaging presentations in 4 patients with advanced HCC (BCLC-C) before (A, C, E, G) and after (B, D, F, H) treatment with apatinib. (A and B) (Case 1), Portal-phase gadolinium-enhanced MR image showing that both a tumor in the liver and an embolus in the vena cava shrank significantly in a PR patient. (C and D) (Case 2), Diffusion-weighted MR imaging showing that intrahepatic metastatic HCC tumors (yellow-circled) disappeared after 4 months of treatment, while metastatic retroperitoneal lymph nodes (blue-circled) shrank dramatically in a PR patient. (E and F) (Case 3), T2-weighted MR image showing significantly decreased tumor activity (yellow-circled) in an SD patient. (G and H) (Case 4), B-ultrasound scan showing a significantly shrunken metastatic infraclavicular lymph node in a PR patient. (I) Changes in tumor size in the longest diameter before and after apatinib treatment (as shown above). 
Table 2: Apatinib treatment-related adverse events

\begin{tabular}{lcc}
\hline Treatment-Related AEs & Any Grade No. (\%) $\mathbf{N}=\mathbf{2 2}$ & Grade $\mathbf{3}$ or $\mathbf{4}$ No. (\%) $\mathbf{N = 2 2}$ \\
\hline Nonhematologic AEs & $18(81.8)$ & $3(13.6)$ \\
hand-foot skin reaction & $17(77.3)$ & $1(4.5)$ \\
Diarrhea & $14(63.6)$ & $6(27.3)$ \\
Hypertension & $13(59.1)$ & $0(0)$ \\
Fatigue & $12(54.5)$ & $1(4.5)$ \\
Hoarseness & $11(50.0)$ & $0(0)$ \\
Rash & $11(50.0)$ & $0(0)$ \\
Decreased appetite & $4(18.2)$ & $0(0)$ \\
Vomiting & $4(18.2)$ & $1(4.5)$ \\
Proteinuria & & \\
Hematologic AEs & $14(63.6)$ & $2(9.1)$ \\
Thrombocytopenia & $13(59.1)$ & $1(4.5)$ \\
Leukopenia & $12(54.5)$ & $1(4.5)$ \\
Neutropenia & $12(54.5)$ & $1(4.5)$ \\
Neutropenia & $7(31.8)$ & $0(0)$ \\
Anemia & & \\
Serum chemistry AEs & $13(59.1)$ & $1(4.5)$ \\
Bilirubin increased & $13(59.1)$ & $0(0)$ \\
ALT /AST increased &
\end{tabular}

Abbreviations: AE, adverse event; ALT, alanine aminotransferase; AST, aspartate aminotransferase.

a decrease in AFP serum levels may be helpful in judging the efficacy of apatinib in advanced HCC therapy.

In this study, thrombocytopenia was found to be another risk factor for TTP. Platelet count can reflect the severity of liver cirrhosis, and is associated with prognosis in $\mathrm{HCC}[5$, $24,25]$. Therefore, it is not surprising that thrombocytopenia increased the overall and tumor-related risk of death in this cohort. Perhaps the fundamental cause of this phenomenon is the effect of cirrhosis on the efficacy of apatinib.

In conclusion, apatinib administration achieved an unprecedented therapeutic effect with an acceptable safety profile in patients with advanced HCC in this small sample size study. Although several limitations may somewhat compromise the reliability of our results (i.e. this being a single center retrospective study, its limited sample size, the potential benefits afforded by combined TACE therapy, and our inability to register mOS), the high PR rate and long mTTP obtained are very impressive. In light of the present results, we believe that it is imperative to design and carry out well-controlled clinical trials to confirm the efficacy of apatinib on advanced HCC.

\section{MATERIALS AND METHODS}

\section{Patients}

From September 14, 2015 to October 17, 2016, 22 patients with advanced HCC received apatinib treatment in the Tianjin Medical University Cancer Institute and Hospital. All patients satisfied the following criteria: Barcelona Clinic Liver Cancer (BCLC) stage C; 18 years of age or older; Eastern Cooperative Oncology Group (ECOG) score 0-2; Child-Pugh classification of liver function A-B; more than 4 weeks of medication time; complete imaging data; followed the imaging requirements of the Response Evaluation Criteria in Solid Tumors (RECIST) 1.1 [26]; and consented to receive apatinib treatment. Patients were diagnosed with $\mathrm{HCC}$ by two imaging modalities, or postoperative pathology, or biopsy.

\section{Therapeutic procedures}

The recommended apatinib dose reported for advanced gastric adenocarcinoma or gastroesophageal junction adenocarcinoma is $850 \mathrm{mg} /$ day orally ${ }^{[8]}$. However, considering that liver cirrhosis and hepatic insufficiency are common among our selected HCC patients, the first 6 patients were given apatinib at $500 \mathrm{mg} /$ day taken $2 \mathrm{~h}$ after dinner. Unfortunately, none of these patients tolerated the serious adverse events that ensued. Thereafter, we implemented a starting dose of $250 \mathrm{mg} /$ day in subsequent patients. Treatment would be interrupted or terminated under the following conditions: serious adverse events, death of patients, or voluntarily giving up. If grade 3 or 4 adverse events occurred during apatinib treatment, we would 
first suspend it for 1-2 weeks to alleviate the side effects; then, apatinib would be continued. Treatment interruptions and up to one dose reductions ( $250 \mathrm{mg}$ every 2 days) were permitted in case of drug-related adverse events. If further dose reductions were required, patients were withdrawn from the study.

\section{Outcomes and assessments}

Blood counts, liver and renal function, and hepatic tumor biomarker tests were performed monthly. MRI or CT scans of measurable lesions were assessed at screening and every 8 weeks thereafter. RECIST version 1.1 was used to assess tumor responses. Disease control was defined as CR, PR, SD, and PD. TTP, ORR, DCR, and OS were used for evaluation of clinical endpoints.

\section{Statistical analysis}

Statistical analyses were performed using SPSS statistical package version 18.0 (SPSS, Chicago, USA). Kaplan-Meier plots were used to estimate the time of HCC progression and survival time. Cox proportional hazard analysis was done to assess the effect of important baseline characteristics on overall survival. HR and 95\% CIs were estimated using a non-parametric log-rank test. $\mathrm{P}$ $<0.05$ was considered as statistically significant.

\section{Abbreviations}

HCC: hepatocellular carcinoma; BCLC: Barcelona Clinic Liver Cancer; CR: complete response; PR: partial response; $\mathrm{SD}$ : stable disease; $\mathrm{PD}$ : progressive disease; TTP: time to disease progression; OS: overall survival; ORR: objective response rate; DCR: disease control rate.

\section{Author contributions}

TZ, HL, and LQ were responsible for the conception and design of the study. TZ and HL were responsible for patient recruitment. YK, LS, ZH, YZ, PC, YC, TS, QL, $\mathrm{TZ}$, and HL contributed to the collection and assembly of data. YK, TZ, HL, and LQ were responsible for data analysis, interpretation, and writing the manuscript. YK and LS contributed equally to this work.

\section{ACKNOWLEDGMENTS}

The authors acknowledge the contributions of the other investigators in this study. This researchwas partly supported by Jiangsu Hengrui Medicine Co., Ltd.

\section{CONFLICTS OF INTEREST}

TZ and HL have had a consultant/advisory role, and received research funding from Jiangsu Hengrui Medicine Co., Ltd. All other authors declared no conflicts of interest.

\section{REFERENCES}

1. Chen W, Zheng R, Baade PD, Zhang S, Zeng H, Bray F, Jemal A, Yu XQ, He J. Cancer statistics in China, 2015. CA Cancer J Clin. 2016; 66: 115-32. https://doi.org/.3322/caac.21338.

2. El-Serag HB, Rudolph KL. Hepatocellular carcinoma: epidemiology and molecular carcinogenesis. Gastroenterology. 2007; 132: 2557-76. https://doi. org/.1053/j.gastro.2007.04.061.

3. Llovet JM, Bruix J. Molecular targeted therapies in hepatocellular carcinoma. Hepatology. 2008; 48: 1312-27. https://doi.org/.1002/hep.22506.

4. Llovet JM, Ricci S, Mazzaferro V, Hilgard P, Gane E, Blanc JF, de Oliveira AC, Santoro A, Raoul JL, Forner A, Schwartz M, Porta C, Zeuzem S, et al. Sorafenib in advanced hepatocellular carcinoma. N Engl J Med. 2008; 359: 378-90. https://doi.org/.1056/NEJMoa0708857.

5. Cheng AL, Kang YK, Chen Z, Tsao CJ, Qin S, Kim JS, Luo R, Feng J, Ye S, Yang TS, Xu J, Sun Y, Liang H, et al. Efficacy and safety of sorafenib in patients in the AsiaPacific region with advanced hepatocellular carcinoma: a phase III randomised, double-blind, placebo-controlled trial. Lancet Oncol. 2009; 10: 25-34. https://doi.org/.1016/ S1470-2045(08)70285-7.

6. Li J, Qin S, Xu J, Guo W, Xiong J, Bai Y, Sun G, Yang Y, Wang L, Xu N, Cheng Y, Wang Z, Zheng L, et al. Apatinib for chemotherapy-refractory advanced metastatic gastric cancer: results from a randomized, placebo-controlled, parallel-arm, phase II trial. J Clin Oncol. 2013; 31: 321925. https://doi.org/.1200/JCO.2013.48.8585.

7. Li J, Qin S, Xu J, Xiong J, Wu C, Bai Y, Liu W, Tong J, Liu Y, Xu R, Wang Z, Wang Q, Ouyang X, et al. Randomized, Double-Blind, Placebo-Controlled Phase III Trial of Apatinib in Patients With Chemotherapy-Refractory Advanced or Metastatic Adenocarcinoma of the Stomach or Gastroesophageal Junction. J Clin Oncol. 2016; 34: 144854. https://doi.org/.1200/JCO.2015.63.5995.

8. Qin S. Apatinib in Chinese patients with advanced hepatocellular carcinoma: A phase II randomized, openlabel trial. Asco Meeting Abstracts,2014,32.

9. Tian S, Quan H, Xie C, Guo H, Lu F, Xu Y, Li J, Lou L. YN968D1 is a novel and selective inhibitor of vascular endothelial growth factor receptor-2 tyrosine kinase with potent activity in vitro and in vivo. Cancer Sci. 2011; 102: 1374-80. https://doi. org/.1111/j.1349-7006.2011.01939.x.

10. Scott AJ, Messersmith WA, Jimeno A. Apatinib: a promising oral antiangiogenic agent in the treatment of multiple solid tumors. Drugs Today (Barc). 2015; 51: 2239. https://doi.org/.1358/dot.2015.51.4.2320599.

11. Hu X, Zhang J, Xu B, Jiang Z, Ragaz J, Tong Z, Zhang Q, Wang X, Feng J, Pang D, Fan M, Li J, Wang B, et al. Multicenter phase II study of apatinib, a novel VEGFR inhibitor in heavily pretreated patients with metastatic 
triple-negative breast cancer. Int J Cancer. 2014; 135: 19619. https://doi.org/.1002/ijc.28829.

12. Ding L, Li QJ, You KY, Jiang ZM, Yao HR. The Use of Apatinib in Treating Nonsmall-Cell Lung Cancer: Case Report and Review of Literature. Medicine (Baltimore). 2016; 95: e3598. https://doi.org/.1097/ MD.0000000000003598.

13. Kou P, Zhang Y, Shao W, Zhu H, Zhang J, Wang H, Kong L, Yu J. Significant efficacy and well safety of apatinib in an advanced liver cancer patient: a case report and literature review. Oncotarget. 2017; 8: 20510-5. https://doi. org/.18632/oncotarget.14724.

14. Ji G, Hong L, Yang P. Successful treatment of advanced malignant fibrous histiocytoma of the right forearm with apatinib: a case report. Onco Targets Ther. 2016; 9: 643-7. https://doi.org/.2147/OTT.S96133.

15. Gnoni A, Santini D, Scartozzi M, Russo A, Licchetta A, Palmieri V, Lupo L, Faloppi L, Palasciano G, Memeo V, Angarano G, Brunetti O, Guarini A, et al. Hepatocellular carcinoma treatment over sorafenib: epigenetics, microRNAs and microenvironment. Is there a light at the end of the tunnel? Expert Opin Ther Targets. 2015; 19: 1623-35. https://doi.org/.1517/14728222.2015.1071354.

16. Hu X, Cao J, Hu W, Wu C, Pan Y, Cai L, Tong Z, Wang S, Li J, Wang Z, Wang B, Chen X, Yu H. Multicenter phase II study of apatinib in non-triple-negative metastatic breast cancer. BMC Cancer. 2014; 14: 820. https://doi. org/.1186/1471-2407-14-820.

17. Song Z, Yu X, Lou G, Shi X, Zhang Y. Salvage treatment with apatinib for advanced non-small-cell lung cancer. Onco Targets Ther. 2017; 10: 1821-5. https://doi.org/.2147/ OTT.S113435.

18. Lencioni R, Llovet JM, Han G, Tak WY, Yang J, Guglielmi A, Paik SW, Reig M, Kim DY, Chau GY, Luca A, del Arbol LR, Leberre MA, et al. Sorafenib or placebo plus TACE with doxorubicin-eluting beads for intermediate stage HCC: The SPACE trial. J Hepatol. 2016; 64: 1090-8. https://doi. org/.1016/j.jhep.2016.01.012.

19. Chen LT, Liu TW, Chao Y, Shiah HS, Chang JY, Juang SH, Chen SC, Chuang TR, Chin YH, Whang-Peng J. alpha-fetoprotein response predicts survival benefits of thalidomide in advanced hepatocellular carcinoma.
Aliment Pharmacol Ther. 2005; 22: 217-26. https://doi. org/.1111/j.1365-2036.2005.02547.x.

20. Shao YY, Lin ZZ, Hsu C, Shen YC, Hsu CH, Cheng AL. Early alpha-fetoprotein response predicts treatment efficacy of antiangiogenic systemic therapy in patients with advanced hepatocellular carcinoma. Cancer. 2010; 116: 4590-6. https://doi.org/.1002/cncr.25257.

21. Tsai MC, Wang JH, Hung CH, Kee KM, Yen YH, Lee CM, $\mathrm{Hu} \mathrm{TH}$, Chen $\mathrm{CH}$, Lu SN. Favorable alpha-fetoprotein decrease as a prognostic surrogate in patients with hepatocellular carcinoma after radiofrequency ablation. J Gastroenterol Hepatol. 2010; 25: 605-12. https://doi. org/.1111/j.1440-1746.2009.06115.x.

22. Personeni N, Bozzarelli S, Pressiani T, Rimassa L, Tronconi MC, Sclafani F, Carnaghi C, Pedicini V, Giordano L, Santoro A. Usefulness of alpha-fetoprotein response in patients treated with sorafenib for advanced hepatocellular carcinoma. J Hepatol. 2012; 57: 101-7. https://doi.org/.1016/j.jhep.2012.02.016.

23. Nakazawa T, Hidaka H, Takada J, Okuwaki Y, Tanaka Y, Watanabe M, Shibuya A, Minamino T, Kokubu S, Koizumi W. Early increase in alpha-fetoprotein for predicting unfavorable clinical outcomes in patients with advanced hepatocellular carcinoma treated with sorafenib. Eur J Gastroenterol Hepatol. 2013; 25: 683-9. https://doi. org/.1097/MEG.0b013e32835d913b.

24. Pang Q, Qu K, Zhang JY, Song SD, Liu SS, Tai MH, Liu HC, Liu C. The Prognostic Value of Platelet Count in Patients With Hepatocellular Carcinoma: A Systematic Review and Meta-Analysis. Medicine (Baltimore). 2015; 94: e1431. https://doi.org/.1097/MD.0000000000001431.

25. Akuta N, Suzuki F, Kobayashi M, Hara T, Sezaki H, Suzuki Y, Hosaka T, Kobayashi M, Saitoh S, Ikeda K, Kumada H. Correlation between hepatitis B virus surface antigen level and alpha-fetoprotein in patients free of hepatocellular carcinoma or severe hepatitis. J Med Virol. 2014; 86: 1318. https://doi.org/.1002/jmv.23790.

26. Eisenhauer EA, Therasse P, Bogaerts J, Schwartz LH, Sargent D, Ford R, Dancey J, Arbuck S, Gwyther S, Mooney M, Rubinstein L, Shankar L, Dodd L, et al. New response evaluation criteria in solid tumours: revised RECIST guideline (version 1.1). Eur J Cancer. 2009; 45: 228-47. https://doi.org/.1016/j.ejca.2008.10.026. 